\title{
Tribological Properties of Poly(methyl methacrylate) Brushes Prepared by Surface-Initiated Atom Transfer Radical Polymerization
}

\author{
Hiroki SaKata, Motoyasu Kobayashi, Hideyuki Otsuka, and Atsushi TaKaharA ${ }^{\dagger}$ \\ Institute for Materials Chemistry and Engineering, Kyushu University, \\ 6-10-1 Hakozaki Higashi-ku, Fukuoka 812-8581, Japan
}

(Received May 16, 2005; Accepted July 4, 2005; Published October 15, 2005)

\begin{abstract}
Surface-initiated living radical polymerization of methyl methacrylate (MMA) using copper/bipyridyl complex was carried out from silicon wafer immobilized with a 2-bromoisobutylate moiety, resulting in the assembly of polymer chains tethered by one end to a surface, a so-called "polymer brush". The thickness of the PMMA brush, which was estimated by ellipsometry and atomic force microscopy, increased linearly with the molecular weight of the chains, and was controlled by the free initiator concentration. The graft density of the PMMA brushes was estimated to be as high as 0.56 chains $/ \mathrm{nm}^{2}$. The frictional properties of the high density PMMA brush were characterized by sliding the stainless ball probe on its surface across a width of $20 \mathrm{~mm}$ at a rate of $90 \mathrm{~mm} / \mathrm{min}$ under loading of $0.49 \mathrm{~N}$ in air at room temperature. The PMMA brush was found to have a lower frictional coefficient and much better wear resistance than the corresponding spin-coated PMMA film because of the anchoring of the chain ends in the brush. In addition, the friction coefficient of the polymer brush significantly decreased in response to soaking in acetone and toluene, and increased in response to immersion in hexane. The tribological properties depended on the solvent quality.

[DOI 10.1295/polymj.37.767]

KEY WORDS Tribology / Friction Coefficient / ATRP / Surface-Initiated Polymerization / Poly(methyl methacrylate) (PMMA) / Polymer Brush / Wear Resistance /
\end{abstract}

Wear-resistant materials with low friction are required for various modern technologies ranging from a hard disk drives to roller bearings in automobile. In particular nonlubricanted moving devices will be increasingly needed for nano-machine technologies in future. To improve the physicochemical properties of solid surfaces, we have been investigating the tribological properties of ultrathin films prepared by chemical vapor adsorption of organosilane compounds with long alkyl chains, which are immobilized on a silicon substrate by means of silyloxy bonds, forming a highdensity monolayer structure. ${ }^{1}$ This immobilized monolayer has a low friction coefficient and great wear resistance, probably because of the low interfacial energy of monolayer surface and strong adhesion between monolayer and substrate. Consequently, the following study focused on a surface-grafted polymer, referred to as a "polymer brush".

Over the last decade, high-density and well-defined polymer brushes have been readily synthesized, since controlled/"living" polymerization techniques ${ }^{2-4}$ have been applied to the surface-initiated polymerization. ${ }^{5-7}$ One of the advantages of living polymerization is quantitative initiation, which means that polymers are propagated efficiently from the initiators on a substrate. Practically, only 1 of 10 initiators bound to the surface is expected to initiate a polymerization because of the steric hindrance of the growing polymer, however, it is sufficient to afford a high-density brush. Atom transfer radical polymerization (ATRP) has been widely employed for the formation of polymer brushes because ATRP is compatible with various functionalized monomers, ${ }^{8-12}$ and the living character of the ATRP process yields polymers with a low polydispersity. Many researchers are investigating the surface properties of tailored polymer brushes,${ }^{13}$ including adhesion, ${ }^{14}$ chromatography, ${ }^{15}$ biomaterials, ${ }^{16}$ wettability, ${ }^{17}$ and biocompatibility. ${ }^{18}$

Although the molecular mechanism of tribology on the brush is still difficult to understand experimentally due to the complexity of friction, lubrication, and wear phenomena, some research groups have tried to analyze the frictional properties of the polymer brush. Klein et al. have found a reduction in the frictional forces between solid surfaces bearing polymer brushes using a newly developed surface force balance, ${ }^{19,20}$ and they have also reported that brushes of a charged polymer (polyelectrolyte) can act as efficient lubricants between mica surfaces in an aqueous medium. ${ }^{21,22}$ Similarly, Osada and his coworkers have reported that the well-defined polyelectrolyte brushes, prepared by controlled radical polymerization using TEMPO, reduce the surface friction of hydrogels in water. ${ }^{23}$ They have also found that the friction

${ }^{\dagger}$ To whom correspondence should be addressed (Tel: +81-92-642-2721, Fax: +81-92-642-2715, E-mail: takahara@cstf.kyushu-u.ac.jp). 


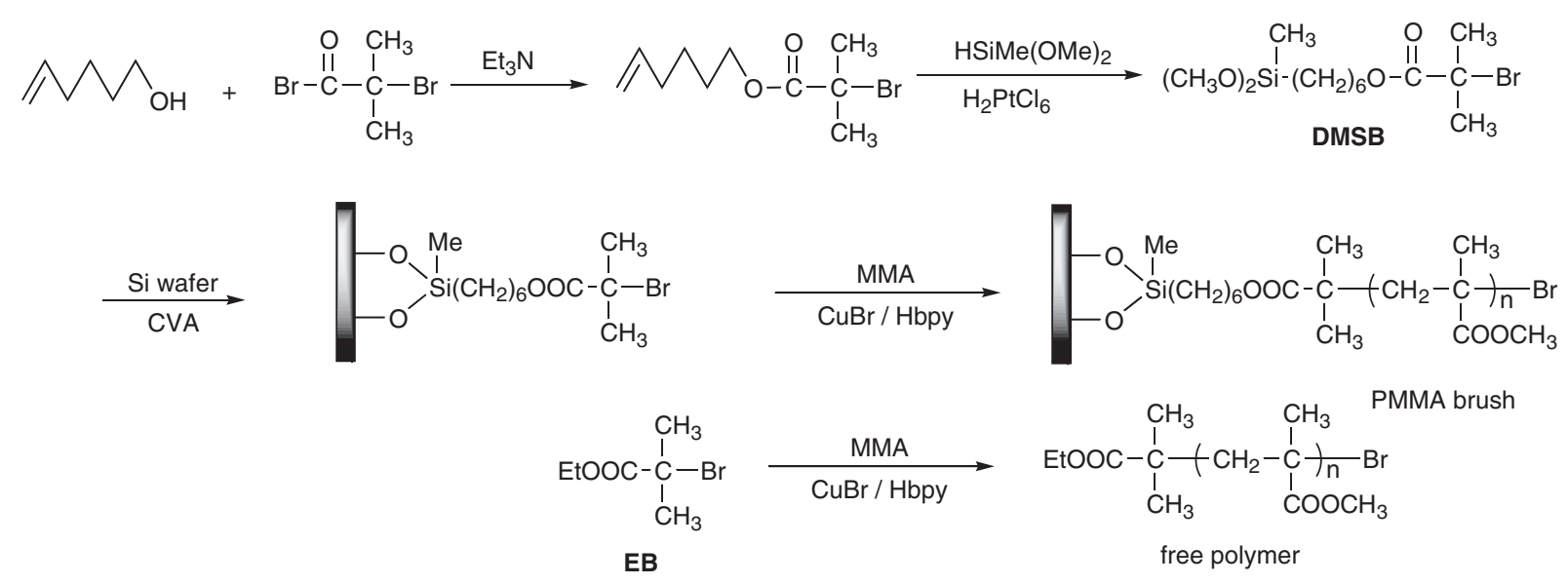

Scheme 1.

depends on the brush length; i.e., gels with longer polymer brushes show higher friction. The lubrication of a polymer brush is interesting from both a scientific and a technological perspective. Tsujii et al. have measured topographic images and force-distance profiles of high-density PMMA brushes by scanning force microscopy (SFM) using a micro silica sphere attached to a cantilever head. ${ }^{24,25}$ The highly anisotropic structure of the swollen brush in toluene revealed extremely strong resistance against compression. These results imply the potential of the polymer brush to serve as a low-friction and a wear-resistant film, although its macro tribology and mechanical properties have not yet been well studied, despite their very importance with regard to practical applications. Therefore we started a project to synthesize tethered polymer brushes on flat silicon substrate by surface-initiated ATRP of MMA, investigating the sliding friction with a stainless probe and the wear resistance of the brush surface under a load of $10^{8} \mathrm{~Pa}$ pressure. The various solvent effects on the tethered brush during sliding friction tests are also described in this paper.

\section{EXPERIMENTAL}

\section{Materials}

Anisole was stirred with sodium tips at $383 \mathrm{~K}$ for $6 \mathrm{~h}$, followed by distillation from sodium under reduced pressure. Copper bromide $(\mathrm{CuBr}$, Wako Pure Chemicals Industries Ltd.) was purified by washing with acetic acid and dried under vacuum. ${ }^{26}$ Methyl methacrylate (MMA) purchased from Wako Pure Chemicals was distilled under reduced pressure over $\mathrm{CaH}_{2}$ before use. 4,4'-Di-n-heptyl-2,2'-bipyridine (Hbpy) was prepared by the dilithiation of 4,4'-dimethyl-2,2'-bipyridine followed by coupling with 1-bromohexane according to the method of Matyjaszewski et $a .^{27}$ Ethyl 2-bromoisobutylate (EB), purchased from Tokyo Chemical Inc., was distilled before use.
Water for contact angle measurements and frictional tests was purified with the NanoPure Water system (Millipore, Inc.). All other reagents were purchased from commercial sources and used as received.

\section{Synthesis of 6'-dimethoxymethyliylyhexyl 2-bromoiso- butylate (DMSB)}

Bromoisobutyl bromide $(51.5 \mathrm{mmol})$ was added dropwise to a stirred solution of 6-undecenyl-1-ol $(51.2 \mathrm{mmol})$ and triethylamine $(71.9 \mathrm{mmol})$ in dry dichloromethane $(85 \mathrm{~mL})$ under a nitrogen atmosphere with cooling in an ice bath, and the solution was then stirred overnight at room temperature (Scheme 1). ${ }^{7}$ After the filtration with suction to remove the hydrochloric salt, the reaction solution was washed with $0.5 \mathrm{~N} \mathrm{HCl}$ and water, and dried over $\mathrm{MgSO}_{4}$. The product was distilled under reduced pressure (321$322 \mathrm{~K} / 0.1 \mathrm{mmHg}$ ) to give 6 '-hexenyl 2-bromoisobutylate as a colorless liquid $(31.9 \mathrm{mmol}, 76 \%)$. The obtained 6'-undecenyl 2-bromoisobutylate $(16.2 \mathrm{mmol})$ and chloroplatinic acid $(0.019 \mathrm{mmol}, 2$-propanol solution) were placed in a three-necked flask, dimethoxymethylsilane $(32.6 \mathrm{mmol})$ was slowly added to the mixture over a period of $30 \mathrm{~min}$. During the reaction mixture was stirred at $323 \mathrm{~K}$ for $12 \mathrm{~h}$, the conversion was checked by NMR. After unreacted dimethoxymethylsilane was removed under a vacuum, the solution was passed through a short column of anhydrous $\mathrm{Na}_{2} \mathrm{SO}_{4}$ to remove the catalyst, and distilled to give $12.8 \mathrm{mmol}(79 \%)$ of $6^{\prime}$-dimethoxymethylslilylhexyl 2-bromoisobutylate (DMSB) as a colorless oily residue. Further purification was not carried out before use. $400 \mathrm{MHz}{ }^{1} \mathrm{H} \mathrm{NMR}\left(\mathrm{CDCl}_{3}\right): \delta 0.1\left(\mathrm{SiCH}_{3}\right), 0.6$ $\left(\mathrm{SiCH}_{2}\right), 1.4\left(-\mathrm{CH}_{2}-\right), 1.7\left(\mathrm{OCH}_{2} \mathrm{CH}_{2}-\right), 1.9\left(\alpha \mathrm{CH}_{3}\right)$, $3.5\left(\mathrm{SiOCH}_{3}\right), 4.2\left(\mathrm{COOCH}_{2}\right) .100 \mathrm{MHz}{ }^{13} \mathrm{C} \mathrm{NMR}$ $\left(\mathrm{CDCl}_{3}\right): \delta-5.7\left(\mathrm{SiCH}_{3}\right), 13.1\left(\mathrm{SiCH}_{2}\right), 22.7\left(\mathrm{SiCH}_{2}-\right.$ $\left.\mathrm{CH}_{2}\right), 25.5\left(\mathrm{SiCH}_{2} \mathrm{CH}_{2} \mathrm{CH}_{2}\right), 28.3\left(\mathrm{OCH}_{2} \mathrm{CH}_{2}\right), 30.9$ $\left(\alpha \mathrm{CH}_{3}\right), 32.8\left(\mathrm{OCH}_{2} \mathrm{CH}_{2} \mathrm{CH}_{2}\right), 50.2\left(\mathrm{SiOCH}_{3}\right), 56.1$ $(\mathrm{CBr}), 66.2\left(\mathrm{OCH}_{2}\right), 171.8(\mathrm{C}=\mathrm{O})$. 
Preparation of Initiator-Immobilized Silicon Substrate

The silicon(111) wafers $(40 \mathrm{~mm} \times 8 \mathrm{~mm})$ were immersed in a mixture of conc. $\mathrm{H}_{2} \mathrm{SO}_{4}$ and $30 \% \mathrm{H}_{2} \mathrm{O}_{2}$ aqueous solution $(70 / 30, v / v)$ at $373 \mathrm{~K}$ for $1 \mathrm{~h}$ to remove the organic contaminant from their surface. Successively, substrates were further cleaned by exposure to vacuum ultraviolet-ray (VUV, $\lambda=172 \mathrm{~nm}$ ) for $5 \mathrm{~min}$ under reduced pressure at $15 \mathrm{mmHg}$ to result in the hydrophilic surface. These silicon wafers and a glass vessel filled with $10 \%$ toluene solution of DMSB were packed in a Teflon container purged with $\mathrm{N}_{2}$ gas, and were allowed to stand in an autoclave at $373 \mathrm{~K}$ for $5 \mathrm{~h}$. During the heating at this temperature, DMSB vapor adsorbed on the surface of the wafers to make an organosilane monolayer, which is known as the chemical vapor adsorption (CVA) method. ${ }^{28,29}$ After these wafers were rinsed with toluene and ethanol, they were dried in vacuo at $373 \mathrm{~K}$ for $10 \mathrm{~min}$ and was stored in a dark place.

\section{Surface-Initiated ATRP}

Typical polymer brush growth was achieved by placing the DMSB-immobilized substrates in a glass tube equipped with a stop cock under argon gas and adding a degassed anisole solution of $\mathrm{CuBr}(0.020$ mmol), Hbpy (0.040 mmol), MMA (50.0 mmol), and ethyl 2-bromoisobutylate (EB, $0.010 \mathrm{mmol}$ ) as a free initiator. The total volume of the polymerization solution was approximately $10 \mathrm{~mL}$. The polymerization solution was degassed by repeating the freeze-andthaw process, and the glass tube was sealed off under the vacuum condition. The polymerization was then allowed to proceed for a set reaction time $(2-24 \mathrm{~h})$ at $363 \mathrm{~K}$, and was terminated by cooling the solution to $273 \mathrm{~K}$ and the addition of a small amount of methanol under ambient pressure. The conversion of MMA was estimated by ${ }^{1} \mathrm{H}$ NMR spectra of the polymerization solution, comparing the relative intensities of the signals due to the unreacted monomer and the produced polymer. The silicon substrates were washed with toluene using a Soxhlet apparatus for $12 \mathrm{~h}$ to remove the free polymer absorbed on their surfaces, and were dried under the reduced pressure at $373 \mathrm{~K}$ for $1 \mathrm{~h}$. The polymer solution was passed through the alumina column using THF to remove catalyst, and was poured into the methanol to precipitate the free polymer. Using the obtained free polymer, spin coat films on silicon wafers were also prepared from toluene solution. The thickness of the spin coat film was adjusted to that of the corresponding polymer brush by changing the spinning rate and concentration of toluene solution.

\section{Measurements}

The number-average molecular weights $\left(M_{\mathrm{n}}\right)$ and molecular weight distribution (MWD) of the free polymer were determined by size exclusion chromatography (SEC) recorded on a Tosoh GPC-8010 system using polystyrene standards calibration, which runs through two directly connected polystyrene gel columns (Shodex GPC KF-804L, $1.0 \mathrm{~mL} / \mathrm{min}$ ) using THF as an eluent at $313 \mathrm{~K}$. The NMR spectra were measured in $\mathrm{CDCl}_{3}$ with a Jeol EX-400 $\left({ }^{1} \mathrm{H} 400\right.$ $\mathrm{MHz}$ ) system. IR spectra were obtained with a Perkin-Elmer Spectra-One KY type (Perkin-Elmer) system coupled with a Mercury Chromium Tell detector. The incident angle of the $p$-polarized infrared beam to the silicon wafer was $73.7^{\circ}$ (Brewstar angle). The thickness of the polymer brush and the spin coat film on the silicon substrate were determined by an imaging ellipsometer (Nippon Laser \& Electronics Lab.) equipped with a YAG laser $(532.8 \mathrm{~nm})$. The polarizer angle was fixed at $50^{\circ}$, and a refractive index of 1.49 was used for the calculations of the film thickness. Atomic force microscopic (AFM) observation was done with SPA 400 with an SPI 3800N controller (Seiko Instruments Industry Co., Ltd.) in air at room temperature, using a $\mathrm{Si}_{3} \mathrm{~N}_{4}$ integrated tip on a commercial triangle $100 \mu \mathrm{m}$ cantilever (Olympus Co., Ltd.) with a spring constant of $0.09 \mathrm{~N} / \mathrm{m}$. XPS measurements were carried out on a PHI ESCA 5800 (PHI Electronics Co., Ltd.) at $10^{-5} \mathrm{~Pa}$ using a monochromatic $\mathrm{Al}-K_{\alpha} \mathrm{X}$-ray source. The contact angles against water were recorded with a drop shape analysis system DSA10 Mk2 (KRÜSS Inc.) equipped with a video camera. The frictional coefficient of the polymer brushes and the cast films were recorded on a Tribostation Type32 (Shinto Scientific Co., Ltd.) by sliding a stainless ball $(\phi 10 \mathrm{~mm})$ on the substrates over a width of $20 \mathrm{~mm}$ at a sliding velocity of $90 \mathrm{~mm} / \mathrm{min}$ under loading of $0.20-0.98 \mathrm{~N}$ at $298 \mathrm{~K}$ (Figure 1). The friction force on the ball probe was transmitted to a stress gauge attached to a probe end, and was recorded automatically. Friction tests in various solvents were also carried out using the polymer brush substrates, which were immersed in the corresponding solvents for $24 \mathrm{~h}$, in advance. The morphologies of the wear trace of the thin films were observed with an S-4300SE

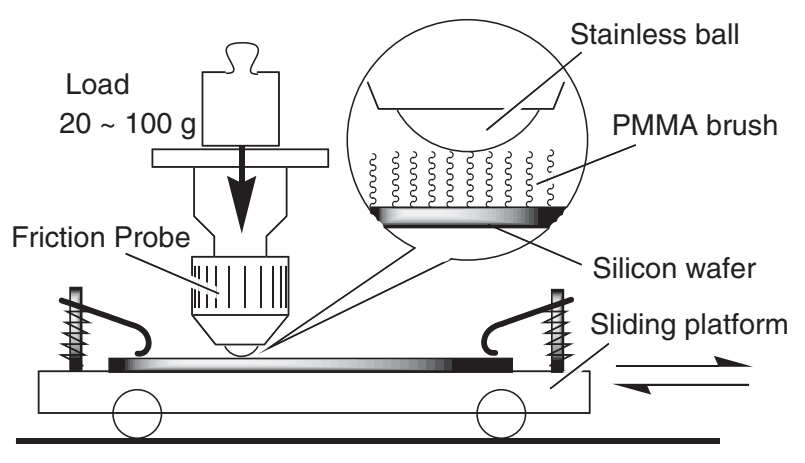

Figure 1. Schematic description of the friction tester setup. 
field-emission SEM (Hitachi Co., Ltd.) equipped with an X-ray microanalysis system (Genesis 7000, EDAX Co., Ltd.) in order to examine the elements on the wear surface.

\section{RESULTS AND DISCUSSION}

\section{Surface-Initiated ATRP of MMA}

The CVA method is often used to prepare the high-density monolayer films of organosilane compounds. ${ }^{30,31}$ Takai et al. have carried out AFM analysis of the monolayer film prepared by CVA, and they found that the monolayer surface is very smooth, with no aggregates and low number of defects. ${ }^{32}$ We prepared here the silicon substrate immobilized with radical initiator, DMSB, by the CVA method. The typical water contact angle of silicon wafer irradiated by VUV is lower than $5^{\circ}$, but water contact angle on the substrate increased to $87^{\circ}$ after the immobilization of DMSB by CVA. XPS spectra of initiator-immobilized silicon wafer showed a carbon signal $\left(\mathrm{C}_{1 \mathrm{~s}}\right)$ at $286 \mathrm{eV}$ associated with the organic portion of the attachable initiator along with the bromide $\left(\mathrm{Br}_{3 \mathrm{~d}}\right)$ signal at $71 \mathrm{eV}$. The $\mathrm{C}_{1 \mathrm{~s}}$ signals attributed to $\mathrm{C}=\mathrm{O}$ and $\mathrm{C}-\mathrm{O}$ bonds were also observed in the narrow scan mode. These results are indicative of formation of a DMSB thin layer on the silicon wafer.

Surface-initiated radical polymerizations of MMA were carried out in the presence of EB as a free initiator coupled with $\mathrm{CuBr}$ and $\mathrm{Hbpy}$ (mole ratio $\mathrm{EB} /$ $\mathrm{CuBr} / \mathrm{Hbpy} / \mathrm{MMA}=1 / 2 / 4 / 1000$ ). Figure 2 shows the plots of $M_{\mathrm{n}}$ and the $M_{\mathrm{w}} / M_{\mathrm{n}}$ index of free polymer produced in the solution as a function of monomer conversion, where the $M_{\mathrm{n}}$ and MWD values were determined by polystyrene-calibrated SEC. The polydispersities of the free polymers were relatively low, and the $M_{\mathrm{n}}$ values were proportional to the monomer conversion, with the slope being very close to the

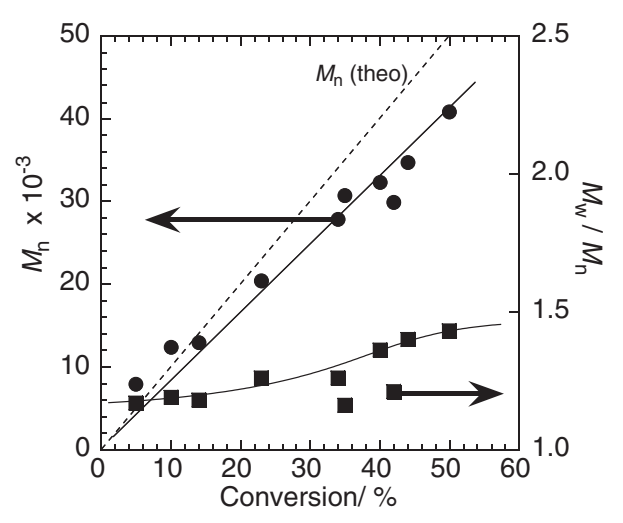

Figure 2. $M_{\mathrm{n}}$ and MWD of free PMMA obtained with EB/ $\mathrm{CuBr} / \mathrm{Hbpy}$ in anisole at $363 \mathrm{~K}:(\bullet) M_{\mathrm{n}}$; (ם) MWD. The dashed line indicates the theoretical value of $M_{\mathrm{n}}$ given by the ratio of $[\mathrm{MMA}] /[\mathrm{EB}]$ and conversion.

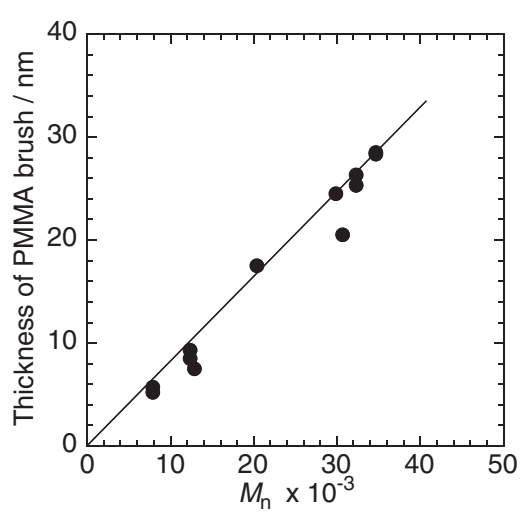

Figure 3. Relationship between brush thickness and $M_{\mathrm{n}}$ of free polymer.

theoretical value. These findings indicate that the controlled polymerization proceeded with a restriction of transfer and termination reactions. The $M_{\mathrm{n}}$ of surfacegrafted PMMA on a silicon wafer cannot be directly determined yet, however, a polymer brush should have the same molecular weight as the value of the corresponding free polymer. ${ }^{24}$ As shown in Figure 3, the thickness of the polymer brushes increased linearly with molecular weight. The thickness of the obtained polymer brush was smaller than the theoretical value of the all-trans conformation given by $0.254 \mathrm{~N}$ and was larger than that of the random coil conformation calculated by $2\left(N b^{2} / 6\right)^{1 / 2}$, where $N$ and $b$ are the degree of polymerization and the statistical segment length of $0.68 \mathrm{~nm}$, respectively. ${ }^{33}$ According to the proportional relationship between the thickness $L_{\mathrm{d}}$ (nm) and $M_{\mathrm{n}}$, the graft density $\sigma$ was estimated to be $c a .0 .56$ (chains $/ \mathrm{nm}^{2}$ ) by following equation,

$$
\sigma=d \cdot L_{\mathrm{d}} \cdot N_{\mathrm{A}} \cdot 10^{-21} / M_{\mathrm{n}}
$$

where $d$ and $N_{\mathrm{A}}$ are the assumed density of bulk PMMA at $293 \mathrm{~K}$ and Avogadro's number, respectively. This graft density is comparatively high, taking into account the volume fraction of the polymer chain. Hence, the tethered PMMA chains would have a relatively extended conformation along the direction normal to the substrate surface. The AFM observation revealed that a homogeneous polymer layer was formed on the substrate. The root mean square (RMS) of the surface roughness was found to be approximately $1.0 \mathrm{~nm}$ in a $10 \times 10 \mu \mathrm{m}^{2}$ scanning area at any location. The water contact angle of PMMA brush was $78^{\circ}$, which was very close to the value $\left(80^{\circ}\right)$ for spin-coated PMMA film. The thickness of the brush did not change in response to repeated rinsing with toluene using a Soxhlet apparatus; therefore, the polymer chains were not physically adsorbed but were chemically anchored on the substrate. Formation of the PMMA brush was also confirmed with XPS and 
Brewstar FT-IR spectra, although the results are not shown here.

\section{Friction Behavior in Dried State}

Dynamic friction tests were carried out by sliding a stainless ball on the substrates at a rate of $90 \mathrm{~mm} / \mathrm{min}$ in air under the normal load ranging from $20-100 \mathrm{~g}$ at room temperature. In the case of a non modified silicon wafer under a normal load of $50 \mathrm{~g}(0.49 \mathrm{~N})$, the theoretical contact area between a stainless probe and substrate can be calculated to be $2.43 \times 10^{-9}\left(\mathrm{~m}^{2}\right)$ by Hertz's theory, ${ }^{34}$ and the average pressure on the contact area was estimated to be $201 \mathrm{MPa}$. Although the actual contact area on the PMMA brush substrate might be larger than the theoretical value, the average pressure supposed to be more than $10^{2} \mathrm{MPa}$. We attempted to demonstrate here whether the polymer brush could resist such a high pressure and friction for industrial application. As shown in Figure 4(a), the dynamic friction coefficient of the polymer brush was found to be $0.20-0.22$ under a normal load of $20 \mathrm{~g}$, and $0.24-0.25$ under a normal load of 50 and $100 \mathrm{~g}$. However, the magnitude of the friction coefficient of the polymer brush was independent of the brush thickness and sliding velocity, ${ }^{35}$ and was almost constant at all normal loads. Large error margins were observed in short brush around 5-20 nm, while the margins of the error bars decreased in size with the thickness of the brush layer, and the friction coeffi-
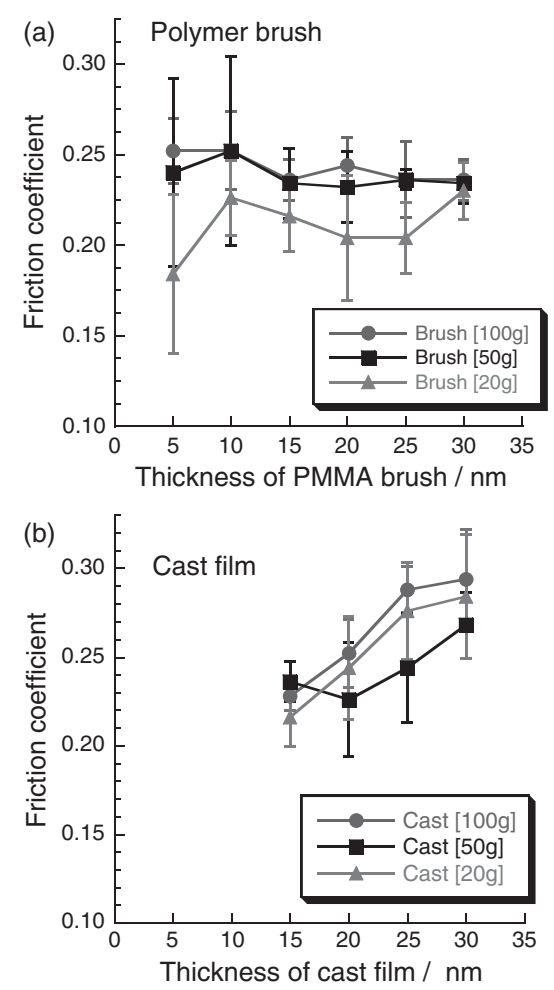

Figure 4. Friction coefficient under dry condition of PMMA brush (a) and cast film (b). cient converged to a constant value. These results indicate that stable sliding was achieved on the substrates, and wear resistance was improved due to the tethered polymer chain end by covalent bonds on the substrate. On the other hand, the dynamic friction coefficient of the cast film increased with film thickness. In addition, a larger friction force was detected than that of brush; for example, the friction coefficients of cast film and brush under loading of $100 \mathrm{~g}$ were 0.31 and 0.24 , respectively. The error margins of the cast films were also larger than those of brush at any film thickness. The thicknesses of these cast films and the molecular weights of spin-coated polymer were almost the same as those of the corresponding polymer brushes; therefore, the grafting structure of polymer brush should contribute to lowering the friction, as described later.

To evaluate the wear resistance of polymer brush, a continuous friction test for $600 \mathrm{~s}$ were performed by sliding a stainless ball on substrates covered with $20 \mathrm{~nm}$ of polymer brush or cast film under a load of $0.49 \mathrm{~N}$ at a rate of $90 \mathrm{~mm} / \mathrm{min}$ (Figure 5). In the early stage of friction test, the friction coefficient of the brush surface increased to 0.5 from 0.25 , but there was no further increase during continuous friction, as shown in Figure 5(a). On the other hand, the friction coefficient of the cast film was gradually
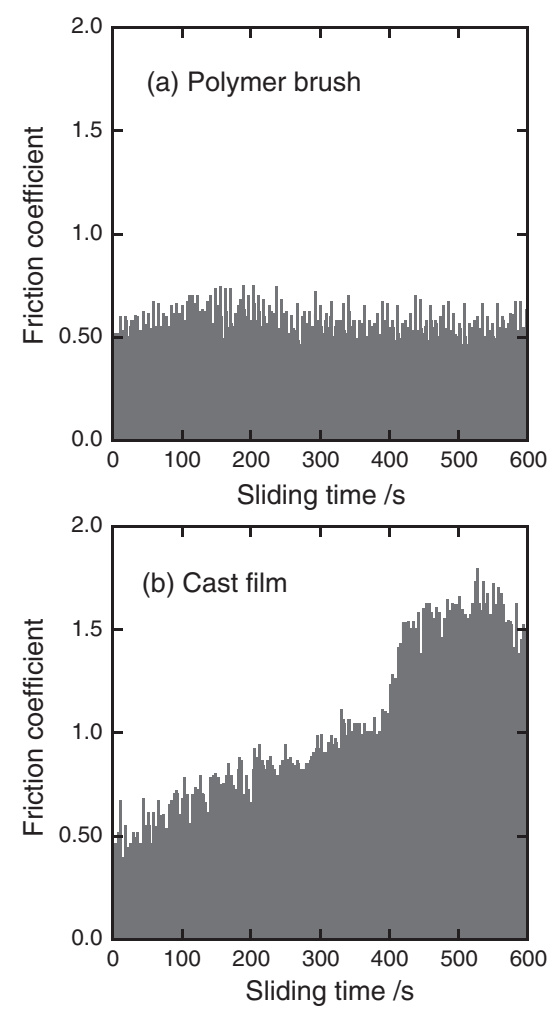

Figure 5. Friction time dependence of friction coefficient on PMMA brush (a) and cast film (b) under a load of $0.49 \mathrm{~N}$ at a sliding velocity of $90 \mathrm{~mm} / \mathrm{min}$ in air: $M_{\mathrm{n}}$ of PMMA $=26000$; Thickness of brush and cast film $=20 \mathrm{~nm}$. 

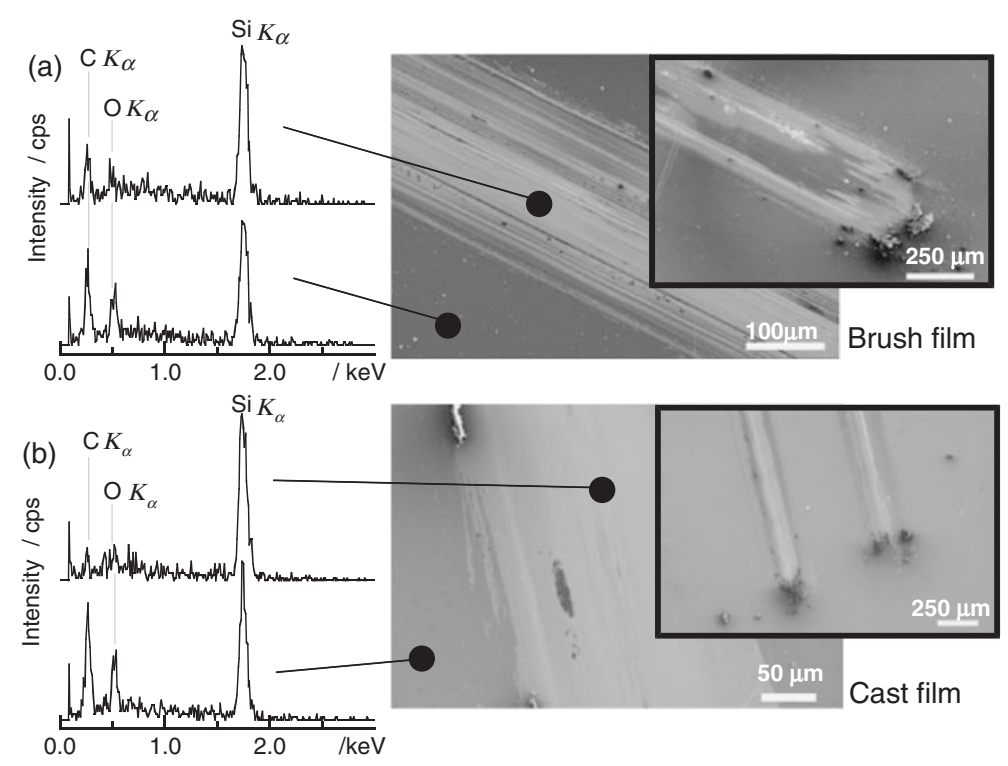

Figure 6. EDX spectra and SEM images of wear track on the PMMA brush (a) and the cast film (b) after the sliding friction test in Figure 5 .

increasing with friction time [Figure 5(b)], and the surface polymer gradually peeled away to form many debris ("wear elements"), some of which were adsorbed on the surface of the probe ("transfer particles") or left on the wear track, interfering with smooth sliding of the probe. A sudden increase of friction coefficient to 1.5 was observed after $400 \mathrm{~s}$, probably because the polymer layer on the wear track had completely peeled off.

The trace scratched by the sliding probe can be seen in the SEM image of wear tracks after the 600-s friction test (Figure 6). No topographic difference in the width and morphology of the scratched tracks could be found between the polymer brush and cast film. An accumulation of wear elements produced by scratching can also be observed at both ends of the sliding trace in both pictures. However, the elemental analysis showed different results. Signals due to carbon $K_{\alpha}$ and oxygen $K_{\alpha}$ could be clearly observed at the point of wear track on polymer brush by energydispersive X-ray (EDX) spectra, which indicated that the PMMA component remained. The observed peak at $1.8 \mathrm{keV}$ attributed to silicon $K_{\alpha}$ must have originated from the silicon substrate. As the matter of course, all peaks attributed with carbon, oxygen, and silicon atoms were observed at the outside of wear tracks. On the contrary, peaks due to neither carbon nor oxygen were observed in the EDX spectrum from the wear track of the spin-cast film. Accordingly, a sliding probe readily scratched the polymer layer on cast film because the polymer molecules on cast film were not anchored on the substrates. In the case of polymer brush, polymer chains were immobilized on the substrates and were highly extended in a perpendicular direction for steric reasons related to the high graft density. ${ }^{36}$ We suppose that such a bound and anisotropic structure restricted the mobility of the endgrafted chain to prevent stretching and scattering by the sliding probe. Of course, some of the brushes were worn away under the strong pressure, as shown in SEM image, although the rest of the tethered components remained during the friction test to result in the low magnitude of friction coefficient.

\section{Effects of Solvent Quality on Friction Behavior}

Since the early stages of polymer brush synthesis, many researchers have suggested that the thickness of polymer brush changes in response to soaking in various solvents; the polymer brush can be stretched in good solvents, and is compressed in poor solvents or when in a dry state on the substrate. Furthermore, the surface morphology and roughness can also be arranged using solvation of polymer brushes. For example, Zhao et al. have reported observing that different surface morphologies of polystyrene-blockPMMA brush on the substrates layer by AFM after treatment with cyclohexane and dichloromethane. ${ }^{37,38}$ The solvent quality affects whether the polymer brush will be stretched or compressed. We next attempted to investigate the influence of solvent quality on the friction of polymer brushes. Friction tests of cast films were not performed because the cast films can be dissolved in organic solvents and removed from the substrates.

The friction coefficients of the PMMA brush with various thickness were measured by sliding a stainless probe under a load of $50 \mathrm{~g}$ at room temperature in acetone as a good solvent, and in $n$-hexane as a poor 

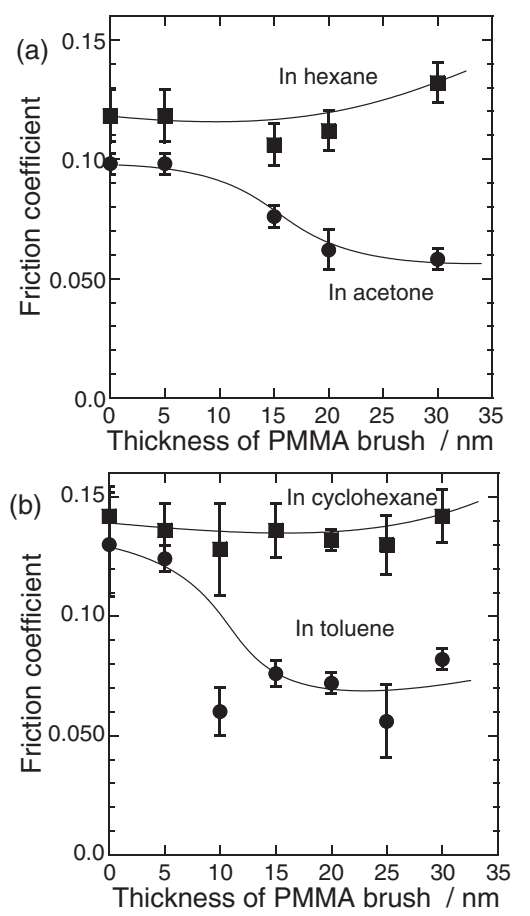

Figure 7. Friction coefficient of the PMMA brush in hexane and acetone (a), in cyclohexane and toluene (b) under a load of $0.49 \mathrm{~N}$ at room temperature.

solvent [Figure 7(a)]. Compared with the friction coefficients of polymer brush in the dry state, the value decreased in both organic solvents due to the fluid lubrication effect. The friction coefficient in acetone was lower than that in hexane, and it decreased with increases in the brush thickness. The friction coefficient of the PMMA brush with a 30-nm thickness was 0.05 , which is a half magnitude of the non modified substrate, suggesting that the polymer in good solvents performs as a good lubricant. In $n$-hexane, however, the friction coefficient of brush with any thickness was almost as the same as that of non modified silicon substrate. This finding suggests that the polymer brushes in poor solvents did not perform well as lubricants, in other words, only fluid lubrication occurred. Similar results were observed in toluene and cyclohexane solution, as shown in Figure 7(b). The dynamic friction coefficient in toluene approached to a value of 0.06 on a $25-\mathrm{nm}$ thickness brush, while the friction coefficient for sliding friction in a cyclohexane system was higher than that in toluene, maintaining a constant value around 0.14 , regardless of the brush thickness. These results suggest that the interaction between the brush surface and the stainless ball (friction probe) was moderated because acetone and toluene were good solvents for PMMA. On the other hand, the PMMA brush surface would be unwilling to be in contact with poor solvent such as hexane and cylcohexane, and prefers to interact with the stainless probe, thus giving a higher friction coefficient.

The wear resistance in solvents was also measured under a normal load of $0.49 \mathrm{~N}$ and a sliding velocity of $90 \mathrm{~mm} / \mathrm{min}$ for $10 \mathrm{~min}$ in toluene and cyclohexane. As we expected, better wear resistance of the polymer brush was observed in toluene compared with that in cyclohexane. Figure 8 shows the SEM image and EDX spectra of the worn surface after the wear-resistance test. The wear track in toluene seems to be slight compared with the scratched surface under the non solvent condition in Figure 6. Almost no accumulation of wear elements was observed at the sides and ends of the sliding trace. Strong peaks due to carbon and oxygen $K_{\alpha}$ were observed in EDX spectra from the worn track surface, indicating that PMMA brush components still covered the substrate surface. We believe that the polymer brush, together with toluene, worked as a lubricant, and reduced the interaction between the stainless probe and the brush. The molecular motion and characteristic structure of polymer brush might impact the wear resistance, especially in a solution. As mentioned above, polymer brush chains in an good solvents typically form an extended structure along the perpendicular direction and show high repulsion against the compression, while the polymer chains tend to be compressed or collapse in poor solvents. ${ }^{39}$ However, brush structures in the presence and absence of a solvent would be squashed by a probe under as high pressure as $10^{2} \mathrm{MPa}$. Therefore, we suppose that the sliding friction and wear resistance

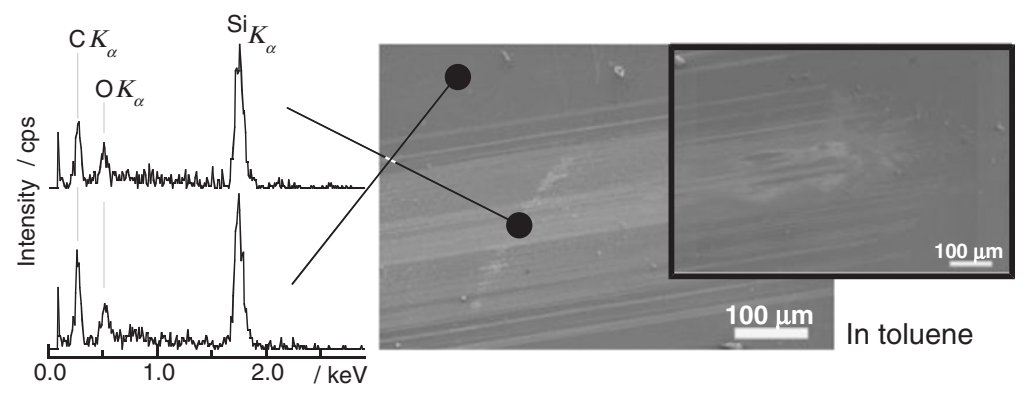

Figure 8. EDX spectra and SEM photograph of wear track on PMMA brush after sliding friction test in toluene under a load of $0.49 \mathrm{~N}$ at a rate of $90 \mathrm{~mm} / \mathrm{min}$ for $600 \mathrm{~s}: M_{\mathrm{n}}$ of PMMA $=28000$; Thickness of brush $=20 \mathrm{~nm}$. 
depend more on the interaction of the brush and probe than the extended structure of polymer brush.

\section{CONCLUSIONS}

The high-density PMMA brushes were obtained by surface-initiated atom transfer radical polymerization using a silicon wafer covered with a flat monolayer of 2-bromoisobutylate derivatives prepared by the CVA method. According to the proportional relationship between the thickness and $M_{\mathrm{n}}$ of the polymer brush, the graft density was estimated to be 0.56 chains $/ \mathrm{nm}^{2}$, which is the so-called high-density brush. The friction coefficient of the polymer brush under a normal load of $0.49 \mathrm{~N}$ in air at room temperature was a lower than that of spin-coated film having the same $M_{\mathrm{n}}$ and thickness as the corresponding polymer brush. The polymer brush revealed better wear resistance than the spin cast film because of the end-grafted structure of the polymer brush. The tribological properties of brush in solution were dramatically changed by the solvent quality. The friction coefficient of PMMA brush in a good solvent such as toluene, decreased further compared with those in air and in a poor solvent such as hexane. In good solvent, polymer brush performed as a lubricant to reduced interaction between the probe and brush surface; as such, the wear resistance was also significantly improved. From these results, it can be concluded that polymer brush prepared by surface-initiated polymerization exhibits excellent tribological properties compared with those prepared by spin-coating.

Acknowledgment. This work was partially supported by a Grant-in-Aid for the 21st century COE Program "Functional Innovation of Molecular Informatics" from the Ministry of Education, Culture, Sports, Science and Technology of Japan. The FESEM observation was performed using S-4300SE (Hitachi Co., Ltd.) at the Collabo-station II, Kyushu University.

\section{REFERENCES}

1. H. Ishida, T. Koga, M. Morita, H. Otsuka, and A. Takahara, Tribology Lett., 19, 523 (2005).

2. K. Matyjaszewski and J. Xia, Chem. Rev., 101, 2921 (2001).

3. C. J. Hawker, A. W. Bosman, and E. Harth, Chem. Rev., 101, 3661 (2001).

4. M. Kamigaito, T. Ando, and M. Sawamoto, Chem. Rev., 101, 3689 (2001).

5. M. Ejaz, S. Yamamoto, K. Ohno, Y. Tsujii, and T. Fukuda, Macromolecules, 31, 5934 (1998).

6. B. Zhao and W. J. Brittain, J. Am. Chem. Soc., 121, 3557 (1999).
7. M. Husseman, E. E. Malmstrom, M. McNamara, M. Mate, D. Mecerreyes, D. G. Benoit, J. L. Hedrick, P. Mansky, E. Huang, T. P. Russell, and C. J. Hawker, Macromolecules, 32, 1424 (1999).

8. T. von Werne and T. E. Patten, J. Am. Chem. Soc., 121, 7409 (1999).

9. M. Ejaz, K. Ohno, Y. Tsujii, and T. Fukuda, Macromolecules, 33, 2870 (2000).

10. C. Perruchot, M. A. Khan, A. Kamitsi, S. P. Armes, T. von Werne, and T. E. Patten, Langmuir, 17, 4479 (2001).

11. W. Huang, J. B. Kim, M. L. Bruening, and G. L. Baker, Macromolecules, 35, 1175 (2002).

12. M. Ejaz, S. Yamamoto, Y. Tsujii, and T. Fukuda, Macromolecules, 35, 1412 (2002).

13. K. Kato, M. Uchida, E.-T. Kang, Y. Uyama, and Y. Ikada, Prog. Polym. Sci., 28, 209 (2003).

14. J. J. Lin, J. A. Silas, H. Bermudez, V. T. Milam, F. S. Bates, and D. A. Hammer, Langmuir, 20, 5493 (2004).

15. X. Huang and M. J. Wirth, Anal. Chem., 69, 4577 (1997).

16. T. Matsuda, M. Kaneko, and S. Ge, Biomaterials, 24, 4507 (2003).

17. A. M. Granville and W. J. Brittain, Macromol. Rapid Commun., 25, 1298 (2004).

18. R. Iwata, P. Suk-In, V. P. Hoven, A. Takahara, K. Akiyoshi, and Y. Iwasaki, Biomacromolecules, 5, 2308 (2004).

19. J. Klein, E. Kumacheva, D. Mahalu, D. Perahia, and L. Fetters, Nature, 370, 634 (1994).

20. R. Tadmor, J. Janik, and J. Klein, Phys. Rev. Lett., 91, 115503 (2003).

21. U. Raviv, S. Giasson, N. Kamph, J.-F. Gohy, R. Jérôme, and J. Klein, Nature, 425, 163 (2003).

22. J. Klein, U. Raviv, S. Perkin, N. Kampf, L. Chai, and S. Giasson, J. Phys.: Condens. Matter, 16, S5437 (2004).

23. Y. Ohsedo, R. Takashina, J. P. Gong, and Y. Osada, Langmuir, 20, 6549 (2004).

24. S. Yamamoto, M. Ejaz, Y. Tsujii, M. Matsumoto, and T. Fukuda, Macromolecules, 33, 5602 (2000).

25. S. Yamamoto, M. Ejaz, Y. Tsujii, and T. Fukuda, Macromolecules, 33, 5608 (2000).

26. T. Grimaud and K. Matyjaszewski, Macromolecules, 30, 2216 (1997).

27. K. Matyjaszewski, T. E. Patten, and J. Xia, J. Am. Chem. Soc., 119, 674 (1997).

28. T. Koga, H. Otsuka, and A. Takahara, Chem. Lett., 31, 1196 (2002).

29. A. Takahara, H. Sakata, M. Morita, T. Koga, and H. Otsuka, Compos. Interfaces, 10, 489 (2003).

30. H. Tada and H. Nagayama, Langmuir, 10, 1472 (1994).

31. K. Hayashi, N. Saito, H. Sugimura, O. Takai, and N. Nagagiri, Langmuir, 18, 7469 (2002).

32. A. Hozumi, Y. Yokogawa, Y. Kameyama, H. Sugimura, K. Hayashi, H. Shinohara, and O. Takai, J. Vac. Sci. Technol., A, 19, 1812 (2001).

33. K. Tanaka, K. Kojio, R. Kimura, A. Takahara, and T. Kajiyama, Polym. J., 35, 44 (2003).

34. If a circle with radius $a(\mathrm{~m})$ is regarded as a contact area between a stainless probe and substrate under a normal load $P(0.49 \mathrm{~N})$, Hertz's theory afford a following relationship using Young's modulus of stainless and silicon wafer, $E_{\mathrm{A}}$ 
$\left(1.96 \times 10^{11} \mathrm{~Pa}\right), E_{\mathrm{B}}\left(1.30 \times 10^{11} \mathrm{~Pa}\right)$, and Poisson's ratio $v_{\mathrm{A}}$ (0.30), $v_{\mathrm{B}}(0.28)$;

$$
\begin{aligned}
& 2 / E=\left(1-v_{\mathrm{A}}^{2}\right) / E_{\mathrm{A}}+\left(1-v_{\mathrm{B}}^{2}\right) / E_{\mathrm{B}} \\
& a=\left(3 / 4 \times 2 / E \times P \times R_{\mathrm{A}}\right)^{3 / 2}
\end{aligned}
$$

where $R_{\mathrm{A}}$ is curvature radius $\left(5.00 \times 10^{-3} \mathrm{~m}\right)$ of stainless ball.

35. Unpublished data. Even if the stainless probe slided on the surface of polymer brush with $20-30 \mathrm{~nm}$ thickness at rate of 90 and $900 \mathrm{~mm} / \mathrm{min}$, the friction coefficients were almost same within error margin.

36. S. Yamamoto, T. Tsujii, and T. Fukuda, Macromolecules, 35, 6077 (2002).

37. B. Zhao, W. J. Brittain, W. Zhou, and S. Z. G. Cheng, J. Am. Chem. Soc., 122, 2307 (2000).

38. S. G. Boyes, W. J. Brittain, X. Weng, and S. Z. G. Cheng, Macromolecules, 35, 4960 (2002).

39. A. Roters, M. Schimmel, J. Ruhe, and D. Johannsmann, Langmuir, 14, 3999 (1998). 